\title{
Retromanía, artificio y transgresión del glam en la música popular española del siglo XXI ${ }^{1}$
}

\author{
Retromania, Artifice and the Transgression of Glam in \\ Spanish Popular Music of the Twenty-first Century
}

Los comienzos del siglo XXI se han distinguido por la masificación de internet y la fragmentación de las audiencias, lo cual ha producido lo que Simon Reynolds (2011) denomina retromanía, es decir, la sucesión e hibridación de diferentes revivals definidos por un sentimiento de nostalgia. El discurso del glam, caracterizado por el énfasis en el artificio y en la transgresión de género, se ha insertado con facilidad en estos revivals debido a su eclecticismo y heterogeneidad musical. El objetivo de este trabajo es analizar cómo la transgresión asociada al glam se adaptó al contexto español en la década del 2000-2010 de manera similar a como se estaba haciendo en el contexto internacional: primero dentro del revival del rock (Babylon Chat, Circodelia) y más tarde recuperando tendencias de los años ochenta como la new wave (Glamour to Kill, Nancys Rubias).

Palabras clave: glam, revival, autenticidad, queer, camp, masculinidad.

The early twenty-first century has been marked by the generalization of Internet and the fragmentation of audiences, which has led to what Simon Reynolds (2011) calls retromania, that is to say, the succession and hybridisation of different revivals defined by a sense of nostalgia. The discourse of glam, characterised by an emphasis on the artifice and transgression of gender, has been included in these revivals with ease due to its eclecticism and musical heterogeneity. The objective of this article is to analyse how the transgression associated with glam was adapted to the Spanish context in the decade 2000-2010 in a similar manner to how this occurred in the international context: initially as part of the revival of rock (Babylon Chat, Circodelia) and later reviving eighties trends such as new wave (Glamour to Kill, Nancys Rubias).

Keywords: glam, revival, authenticity, queer, camp, masculinity.

\section{El glam, la retromanía y su adaptación al mercado musical español}

La música popular de la primera década del siglo XXI se distingue por un sentimiento de nostalgia que provoca la sucesión e hibridación de diferentes revivals, lo que Simon Reynolds denomina retromanía ${ }^{2}$. Así, diferentes artistas y bandas trataron de volver al discurso del rock en el

${ }^{1}$ El presente artículo está inscrito en el Proyecto de Investigación Músicas en conflicto en España y Latinoamérica: entre la hegemonía y la transgresión (siglos XX y XXI) (HAR2015-64285-C2-1-P), coordinado por el Grupo de Investigación en Música Contemporánea de España y Latinoamérica Diapente XXI (GIMCEL) de la Universidad de Oviedo y ha sido financiado a través del Programa Severo Ochoa de Ayudas Pre-doctorales para la Investigación y Docencia del Principado de Asturias.

2 Simon Reynolds: Retromania: Pop Culture's Addiction to Its Own Past, Londres, Faber and Faber, 2011. 
que este era sinónimo de juventud, rebeldía y exceso, y entendido como expresión folk con capacidad para articular una comunidad a través de la demostración de integridad 3 -lo que Allan Moore denomina "autenticidad en primera persona" -. A partir del 2004 también se comenzaron a recuperar tendencias de los ochenta como la new wave -caracterizada por su modernidad posmoderna y eclecticismo (caso de The Killers)- o el postpunk (por ejemplo, la banda Interpol) ${ }^{5}$. El glam ha sido adaptado de diferentes maneras en algunos de estos revivals (The Killers, The White Stripes, Antony and The Johnsons) y la etiqueta glam se ha utilizado para aludir al artificio, la visualidad y la transgresión de género.

El glam es una corriente del rock que se desarrolló durante la primera mitad de los años setenta en los países anglosajones -fundamentalmente en el Reino Unido, pero también de forma minoritaria en Estados Unidosy que englobaba a artistas y bandas muy diversas en sus propuestas sonoras, como David Bowie, T. Rex, Roxy Music o los New York Dolls. A pesar de su influencia constante en el rock y en el pop, el glam presenta problemas en su definición: así, con frecuencia se ha aludido para ello a su énfasis en el plano visual, con una estética que explota la androginia y la extravagancia en detrimento de la música ${ }^{6}$. Philip Auslander ha estudiado en profundidad la corriente en Performing Glam Rock: Gender and Theatricality in Popular Music ${ }^{7}$, desde una perspectiva multidisciplinar enmarcada dentro de los estudios sobre performance. Auslander explica que la visualidad del glam de los setenta chocaba con la anti-ocularidad del rock, que está ligada a la construcción de la autenticidad y el deseo de ser percibido como discurso contracultural opuesto a la industria del entretenimiento y el espectáculo, a la que consideraban una herramienta del capitalismo ${ }^{8}$. A lo largo de las décadas el término glam se ha utilizado para hacer referencia a propuestas - no solo musicales- en las que la parte visual focaliza la atención del espectador debido a su extravagancia, manierismo y manifiesta artificialidad9. Es precisamente esta exageración estética, este

\footnotetext{
${ }^{3}$ Simon Frith: "The Magic that Can Set You Free': the Ideology of Folk and the Myth of the Rock Community", Popular Music, 1, 1981, pp. 159-168.

${ }^{4}$ Allan F. Moore: "Authenticity as Authentication", Popular Music, 21, 2, 2002, pp. 209-223: 211-214.

${ }^{5}$ Theo Cateforis: Are We not New Wave?: Modern Pop at the Turn of the 1980s, Ann Arbor, University of Michigan Press, 2011; Juan Manuel Corral (ed.): New Wave \& Post-punk (1978-1984): de Depeche Mode a Franz Ferdinand, Madrid, T\&B Editores, 2008, pp. 313-347.

${ }^{6}$ Catherine Charlton: Rock Music Styles: a History, Boston, McGraw-Hill, 1998, p. 151; Roy Shuker (ed.): Key Concepts in Popular Music, Londres, Routledge, 1998, p. 177.

${ }^{7}$ Philip Auslander: Performing Glam Rock: Gender and Theatricality in Popular Music, Ann Arbor, University of Michigan Press, 2006.

${ }^{8}$ P. Auslander: Performing Glam..., pp. 15-19.

9 Ibid., pp. 232.
} 
dandismo ${ }^{10}$, el que ha levantado la suspicacia de las audiencias del rock, identificadas con la autenticidad y los estereotipos roqueros de masculinidad.

Musicalmente el glam es heterogéneo y no responde a unos patrones o estructuras sonoras definidas. De hecho, el término específico glam rock se ha usado para denominar una pequeña parte del glam británico de los setenta que no era un género en sí mismo, sino un revival del rocanrol de los cincuenta ${ }^{11}$. Sin embargo, a pesar de que autores como Cagle afirman que el glam musicalmente es incoherente o un "anti-género"12, las tendencias que lo adaptan utilizan géneros (britpop/pop, rocanrol; glam metal/lite metal; new romantics/synth pop; etc.) que responden a unas estrategias comunes, a veces conectadas con las propuestas de Andy Warhol y el pop art (amateurismo, comercialidad, uso de los mass media, sensibilidad camp, etc.)

En España el glam llegó durante los últimos años de la dictadura franquista, debido a la cual no pudo tener más que una adaptación minoritaria y de carácter underground -fundamentalmente por los grupos Brakaman y Burning-13. Por ejemplo, Jaime Stinus (exguitarrista de Brakaman) explica que: "El rock que hacíamos en Brakaman estaba encuadrado dentro del glam, lo cual en la España de mediados de los setenta era una completa locura, pues casi nadie lo entendía [...] la gente decía que eso era música de maricones..."14. Asimismo, Eduardo Haro Ibars (1948-1988) publicó Gay Rock en 1975, el primer libro sobre el glam en español e imprescindible para iconos de la Movida ${ }^{15}$ como Alaska, quien declaró: "Cuando leí el libro de Eduardo Haro Ibars tenía muy claro que yo quería formar parte de ese mundo, quería ser como David Bowie, Lou Reed y Marc Bolan"16. Así, durante la transición democrática y en los ochenta, el glam fue una de las principales herramientas de transgresión en propuestas como las de la Orquesta Mondragón, Tino Casal o Almodóvar \& McNamara, que pusieron de manifiesto la nueva situación de libertad, que les permitía exhibir una sexualidad deliberadamente ambigua y andrógina. En la primera década del siglo XXI artistas y bandas españolas como Nancys Rubias o

\footnotetext{
10 Stan Hawkins: The british Pop Dandy: Masculinity, Popular Music and Culture, Farnham, Ashgate, 2009, pp. 31-35.

11 P. Auslander: Performing Glam..., pp. 51.

12 Van M. Cagle: Reconstructing Pop / Subculture: Art, Rock and Andy Warhol, Thousand Oaks, Sage Publications, 1995, p. 222.

${ }^{13}$ Eduardo Haro Ibars: Gay Rock, Madrid, Ediciones Júcar, 1975, pp. 121-125.

14 Salvador Domínguez: Los hijos del rock: los grupos hispanos 1975-1989, Madrid, Sociedad General de Autores, 2004, p. 311.

${ }^{15}$ La Movida es un fenómeno cultural madrileño que se desarrolló durante la primera mitad de los ochenta y que se difundió después a otras ciudades españolas. Englobaba tanto a grupos musicales (Alaska y los Pegamoides, Radio Futura, Nacha Pop, Aviador Dro, etc.) como a integrantes de otras artes como el cine (Pedro Almodóvar) o la pintura (Las Costus), y se considera producto de la apertura al exterior y la libertad de que disfrutaba el país tras la caída del franquismo.

16 Jesús Ordovás: La revolución pop, Madrid, Celeste, 2002, p. 28.
} 
Circodelia, adaptando los procesos de revival al contexto nacional, insertaron algunas de las estrategias del glam en su discurso, demostrando que su transgresión es capaz de funcionar de forma eficaz también fuera del ámbito anglosajón. El objetivo de este trabajo es analizar dichas estrategias y observar qué similitudes y particularidades presentan los grupos españoles con respecto a sus homólogos extranjeros.

\section{Bailando con Babylon Chat}

Babylon Chat se formaron a mediados de los noventa en Asturias y tuvieron una destacada carrera dentro de las escenas underground e incluían el glam en su discurso. Los componentes más destacados de la banda eran su líder Igor Paskual (voz), Héctor Tuya (guitarra) y Ricardo Saavedra (bajo). La formación grabó Sex Shop (Boomerang Records) en 1997. En él ya se podían apreciar las líneas generales de su discurso, que adoptaba elementos visuales del glam (estampados de leopardo, maquillaje) a través de una estética oscura y agresiva (pintalabios y sombras de ojos negras, ropas de cuero, collares de tachuelas, etc.) Sin embargo, no fue hasta la grabación de Hotel Adicción en el 2000 con la compañía Avispa que lograron obtener atención fuera de los circuitos locales. Así, un año más tarde produjeron el que fue su trabajo más celebrado, Bailando con Brando (Avispa), con el que alcanzaron difusión a nivel naciona ${ }^{17}$. El grupo comenzó a colaborar entonces con el cantante Loquillo (José María Sanz), quien grabó con ellos una versión del tema Vicious de Lou Reed y los convirtió en teloneros de su gira de presentación de Cuero español ${ }^{18}$. A partir de entonces, Igor Paskual empezó a componer para él, trabajo que combinó durante un tiempo con su participación en la banda, hasta que la abandonó y dejó el liderazgo en manos de su guitarrista Héctor Tuya. Con esta nueva plantilla, Babylon Chat grabaron una versión de Like an Hurricane de Neil Young en 2004 y el álbum Baile de disfraces (Iberautor Promociones Culturales) en 2005, disolviéndose poco después de su publicación.

Desde la perspectiva de la primera década del 2000, el glam es considerado parte del canon y de la tradición clásica del rock, por lo que su adaptación es un elemento potencial de autenticidad para las bandas nacionales y extranjeras. Por ello, Babylon Chat citaban como influencias foráneas el rocanrol de los cincuenta y artistas y grupos de glam rock -como Slade, T. Rex o David Bowie- junto a referentes del rock nacional que ya habían adaptado previamente estos discursos (Loquillo, en el caso del rocanrol, y

\footnotetext{
${ }^{17}$ Juanjo Ordás: "Operación Rescate: Babylon Chat", Efe Eme, 24, diciembre, 2009.

${ }_{18}$ Igor Cubillo: "Igor Paskual: 'No es que esté en el Sporting de Gijón y quiera ascender al Barcelona"', Efe Eme, diciembre, 2002, pp. 76-77.
} 
Burning, en el caso del glam). Así, su cantante Igor Paskual explicaba que para ellos Burning y Loquillo "supieron combinar siempre lo que son las influencias extranjeras, de bandas como New York Dolls, los Clash o los Rolling Stones, adaptadas a la realidad y al idioma" de España ${ }^{19}$.

Babylon Chat utilizaron el revival del rocanrol en temas como Camaleón, Glamour o Dandy, en los que realizaban, además, referencias textuales a la corriente -por ejemplo, en el estribillo de Camaleón se aludía a David Bowie y su personaje de Ziggy Stardust (Ziggy "polvo de estrellas") en los versos: "Camaleón, el maquillaje te hace especial [...] polvo estelar en tu gracia animal". Igualmente, usaron el punk y el hard rock (como en su día hicieron algunos referentes del glam, como los New York Dolls o Alice Cooper) para construir un discurso más agresivo, relacionado con la performance de su masculinidad (como por ejemplo en Perra de amor).

El grupo incluía también elementos asociados con la alteridad y la transgresión de género del glam en el plano visual (estampados de leopardo, boas de plumas - exotismo-, prendas de cuero -sadomasoquismo-, maquillaje "monstruoso", etc.), y en la puesta en escena (baile). Estos rasgos que en el glam anglosajón de los setenta entraban en conflicto con la autenticidad por su carácter de actuación preparada, asociada con la falta de espontaneidad o naturalidad, y por su alto grado de artificialidad- son percibidos y adaptados en el siglo XXI como revival del género e interpretados como parte de la imaginería clásica (es decir, "auténtica") del rock. Por ello, el anuncio del lanzamiento de Bailando con Brando en la revista Efe Eme-que llevaba por título "Suscríbete y vuelve al glam" -redundaba en esta idea afirmando que "bajo el maquillaje y la pose glam" Babylon Chat eran "unos convencidos seguidores del mejor rock [...] escrito en castellano" y que el disco constituía un "tratado de buen rock and roll"20.

\section{El glam y la construcción de la autenticidad}

La interpretación del glam como parte del canon y la historia del rock permitió a Babylon Chat absorber, por una parte, el mito de autenticidad para incluirse dentro de las escenas del rock y, por otra, enfatizar ciertos aspectos que entraban en conflicto con la otra propuesta alternativa local el Xixón Sound-, influida por el grunge y géneros cuya veracidad era construida de forma diferente ${ }^{21}$. Las alusiones al glam en Babylon Chat son constantes tanto en la prensa como en las declaraciones del grupo: por

\footnotetext{
${ }^{19}$ Ibid., p. 77.

20 "Suscríbete y vuelve al glam", Efe Eme, marzo, 2002, p. 51.

${ }^{21}$ Por ejemplo, eludiendo la demostración de artificialidad visual, utilizando ropa corriente -como las denominadas camisas "de leñador"- como signo de sinceridad y de identificación con la audiencia.
} 
ejemplo, Igor Cubillo en Efe Eme comenta que la banda canta "en castellano, con la consiguiente doble dosis de satisfacción para quienes disfrutan con sus riffs bien definidos [...] su toque altanero y unas capas de maquillaje que les permiten pavonearse como cabecillas del glam español"22. Sin embargo, se reitera la idea de que a pesar de la exageración estética son simplemente una banda de rock, para la que el glam es un elemento de provocación (sobre todo en su directo) y cuya visualidad no entraba en conflicto con su profesionalidad (y autenticidad) musical. Así, el guitarrista Héctor Tuya admitía: “[...] por supuesto, sí tenemos referencias evidentemente glamies, con un look muy provocador y una puesta en escena muy en esa línea, pero creo que lo nuestro es el rock and roll clásico"23.

Philip Auslander en Liveness: Performance in a Mediatized Culture explica cómo es precisamente a través del directo que los grupos de rock se legitiman haciendo que los discos imiten la performance para demostrar que los músicos que tocan en concierto son los auténticos - es decir, los mismos que suenan en la grabación-24. Esta estrategia es utilizada por Babylon Chat, quienes no sólo pagan su deuda con la audiencia realizando conciertos regularmente y en ámbitos locales minoritarios (underground), sino que además producen música en géneros que se pueden reproducir fielmente en directo (como el rocanrol, el punk, el garage y el hard rock). De hecho, el crítico Juanjo Ordás declara que la gran virtud de Bailando con Brando es su "sabor clásico" logrado "mediante una producción siempre limpia", tanto que "podría tratarse de un disco en directo" 25.

Esta interpretación del glam como rock clásico con una historia y una tradición se opone al discurso del Xixón Sound, cuyas bandas (por ejemplo, Australian Blonde) enfatizaban sus influencias extranjeras utilizando el inglés ${ }^{26}$, al contrario que Babylon Chat, quienes cantaban en español. Además, Babylon Chat reaccionaban contra la seriedad del Xixón Sound de manera similar a como hacía el glam británico en los setenta con la contracultura $^{27}$, recuperando el discurso en el que el rock se identifica con la juventud y expresa la rebeldía, la energía, la sexualidad y el deseo de diversión propios de la adolescencia ${ }^{28}$. Así, Héctor Tuya opinaba que ellos

\footnotetext{
22 Igor Cubillo: "Bailando con Brando", Efe Eme, marzo, 2002, p. 54. p. 14

${ }^{23}$ Fernando Íñiguez: "Babylon Chat: desde Asturias con glamour y chulería", Efe Eme, enero, 2001,

${ }^{24}$ Philip Auslander: "Tryin' to Make it Real: Live Performance, Simulation, and the Discourse of Authenticity in Rock Culture", Liveness: Performance in a Mediatized Culture, Nueva York, Routledge, 1999, pp. 73-127: 88 .

25 Juanjo Ordás: "Operación Rescate: Babylon Chat", Efe Eme, 24, diciembre, 2009.

26 Pablo Gil: Guía de música independiente en España, Madrid, Ediciones Vosa, 1998, pp. 21-22.

${ }^{27}$ P. Auslander: Performing Glam..., pp. 9-19.

${ }^{28}$ Lawrence Grossberg: We Gotta Get out of this Place: Popular Conservatism and Postmodern Culture, Londres-Nueva York, Routledge, 1992, p. 205.
} 
eran divertidos en comparación con estas otras bandas, ya que no tenían "nada de ese ensimismamiento típico del grunge" cuyos seguidores mostraban una actitud de "estoy de puta madre pero soy un chico atormentado" 29.

\section{El glam y la performance de la heterosexualidad}

Siguiendo el discurso tradicional del feminismo, Judith Butler analiza cómo las identidades de género no tienen un fundamento biológico, sino que son creadas, performadas y fijadas a través de la repetición ${ }^{30}$. Philip Auslander explica que la autenticidad del rock es también un mito performado, por lo que ambos discursos se construyen y se interrelacionan ${ }^{31}$. Así, es posible percibir cómo, por ejemplo, la construcción de autenticidad del llamado cock rock está unida a la idea de la masculinidad heterosexual, asociada con la fuerza física y el control $^{32}$, mientras que géneros como el glam, que la desafian y reinterpretan, juegan con las identidades sexuales y la ambigüedad. Babylon Chat siguen la estela de iconos como Mick Jagger (cock rock) o Iggy Pop (proto-punk glam) quienes, a través de la performance del deseo sexual en la esfera pública, crean un estado de tensión y de confrontación que persigue la provocación o el shock del público ${ }^{33}$. Así, por ejemplo, durante la actuación de Babylon Chat en el programa televisivo Plan 9, la última canción es simplemente un solo instrumental cuyo objetivo es servir de fondo para la escena en la que el cantante se rocía de manera sensual con el contenido de una botella de champán ${ }^{34}$.

La masculinidad en la banda se asocia con la potencia y la energía física (sexual), por lo que, al ser excesiva, el sexo es planteado como un acto de temeridad y el género femenino como una fuente peligrosa de inestabilidad: van, como indica el segundo tema de Bailando con Brando, con demasiada energía ("demasiado deprisa"), a un lugar demasiado peligroso ("demasiado salvaje"). Así, Babylon Chat se refieren al calor del deseo sexual y a su personificación en el fuego para indicar excitación y peligrosidad: por ejemplo, en la portada de Hotel Adicción en la que incluyen llamas.

\footnotetext{
${ }^{29}$ F. Íñiguez: "Babylon Chat ...", p. 14.

30 Judith Butler: Gender Trouble: Feminism and the Subversion of Identity, Nueva York, Routledge, 1990, pp. 24-25.

31 P. Auslander: "Tryin' to...", p. 84.

32 S. Frith, Angela Mac Robbie: "Rock and Sexuality", Screen Education, 29, 1978, pp. 3-19.

33 Steve Waksman: "Death Trip: Alice Cooper, Iggy Pop and Rock Theatricality", This Ain't the Summer of Love: Conflict and Crossover in Heavy Metal and Punk, Berkeley, University of California Press, 2009, pp. 70-103.

34 Babylon Chat: "BABYLON CHAT EN PLAN 9", Plan 9, https://www.youtube.com/watch?v= HdA7efnIDws (última consulta: 15-1-2016).
} 


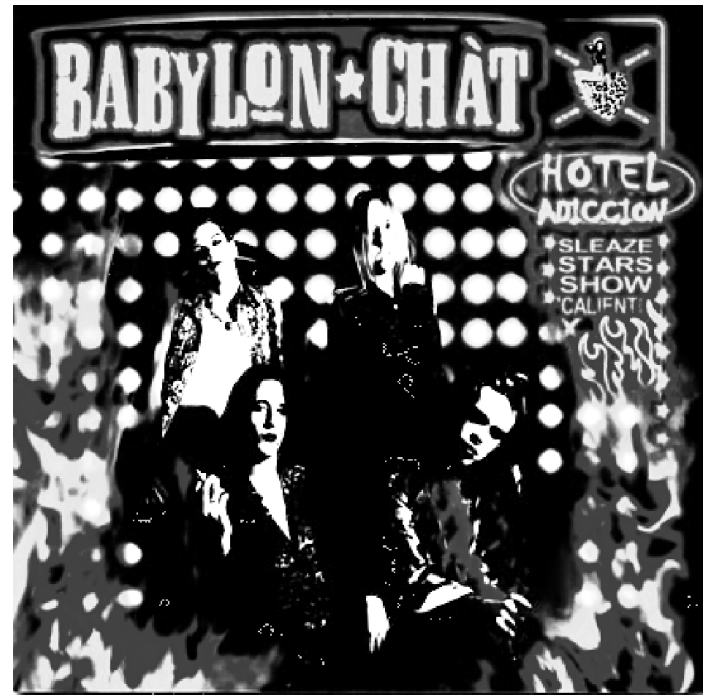

Portada del álbum Hotel Adicción de Babylon Chat (Avispa, 2000)

Antes que ellos, Burning editaron un single en 1974 titulado Estoy ardiendo (I'm Burning) que redundaba en la misma idea. Esta explosión descontrolada de energía se despliega plenamente en el directo, donde la sexualidad es un elemento performativo más, utilizado para generar provocación y conectado con la estética del exceso del rock descrita por Grossberg ${ }^{35}$.

Esta escenificación, en la que el hombre se convierte en objeto de deseo y pasa a ser el sujeto pasivo al que se mira, subvertiría los roles tradicionales de género en los que, según Laura Mulvey ${ }^{36}$, es la mujer la que ha de ser contemplada, pudiendo generar homofobia al ubicar al sujeto en una posición invertida, asociada al otro género: de hecho, Igor Paskual en su libro El arte de mentir relata cómo un muchacho quería enfrentarse con él, "en teoría", por su "vida promiscua" y "correrías nocturnas con chicos", y porque "en su opinión" los miembros de Babylon Chat eran "todos una "pandilla de maricones" "37. De esta forma, Babylon Chat, al igual que otros como los Rolling Stones o Burning sexualizan tanto su masculinidad que acaban por dirigirse promiscuamente a ambos $\operatorname{sexos}^{38}$. Este discurso demuestra de forma implícita que si la masculinidad (aunque sea la asociada a la heterosexualidad) se puede performar en exceso es que, efectivamente, no es una identidad natural, sino que está construida y generada artificialmente. Babylon Chat al identificarse con atributos asociados al estereotipo tradicional del hombre (potencia, energía / fuerza fisica) a pesar de su promiscuidad y su teatralizada exageración seguirían percibiéndose como masculinos, no poniendo en cuestión sino enfatizando su condición de "auténtica" banda de rock.

\footnotetext{
${ }^{35}$ L. Grossberg: We Gotta..., pp. 206 y 207.

36 Laura Mulvey: "Visual Pleasure and Narrative Cinema", Screen, 16, 3, 1975, pp. 8-18.

${ }^{37}$ Igor Paskual: El arte de mentir, Valladolid, Difácil, 2013, p. 72.

38 Sheila Whiteley: "Little Red Rooster Vs. the Honky Tonk Woman: Mick Jagger, Sexuality, Style and Image", Sexing the Groove: Popular Music and Gender, Sheila Whiteley (ed.), Londres, Routledge, 1997, pp. 67-95, 67 .
} 


\section{Las máquinas románticas de Circodelia: el glam y el canon}

Los madrileños Circodelia fueron un grupo ecléctico distinguido por el uso de la ironía y el revival, y por haber sido uno de los que más fielmente adaptaron el glam rock británico de los setenta al contexto español. La banda se creó en 1996, inicialmente con el nombre de Tono Voodoo, y sus componentes más destacados eran Víctor Pérez (cantante), Pablo Parser y Miguel Íniguez (guitarras y parte compositiva). En 1999 ganaron el concurso Jóvenes Creadores y un año más tarde el deVilla de Madrid, con el cual adquirieron mayor visibilidad. Así, fueron contratados poco después por la compañía Pep's Records, que grabó sus dos primeros álbumes: Circodelia, en 2002 (producido por Alejo Stivel), y Lo trágico es magnético, en $2003^{39}$. La amplia difusión y recepción del single Las chicas de las canciones, del que se vendieron cerca de 15.000 ejemplares $^{40}$, puso en cuestión la autenticidad del grupo para ciertos sectores de la audiencia, que los consideraron excesivamente comerciales. Finalmente, en 2006 grabaron su último disco, Máquinas Románticas, con la compañía Tool Music.

El eclecticismo retro de Circodelia se construye de forma intertextual a través del texto musical (uso e hibridación de diferentes estilos -rocanrol, hard rock, salsa, etc.), de sus paratextos (iconografia de las portadas) y de sus metatextos (declaraciones sobre sus referentes estéticos) ${ }^{41}$. Matthew Bannister argumenta que algunas de las estrategias del indie guitar rock de los ochenta -aplicables también a géneros alternativos como el britpop o el grunge- eran, por un lado, el archivalismo o énfasis en el coleccionismo de música y, por el otro, la obsesión por la creación de un canon de la música popular ${ }^{42}$. De este modo, las bandas ya no sólo necesitaban un cantante sexy o un virtuoso, sino también de un historiador del pop, y llegaban incluso a tratar de educar al público ${ }^{43}$. Sin embargo, hablar sobre las influencias puede comprometer la originalidad de los músicos, por lo que la canonización y el archivalismo, aun siendo esenciales para entender las escenas del indie, son también problemáticos porque comprometen su creatividad ${ }^{44}$. Por ello, el uso ecléctico del revival sonoro es uno de los puntos conflictivos para Circodelia en sus aspiraciones de ser percibidos como auténtica banda de rock, no solo por sus alusiones a propuestas alternativas

\footnotetext{
${ }^{39}$ Isabel Aguilar: "Circodelia: 'No nos matamos por conseguir un disco de oro", ABC, 26-II-2004, p. 84 .

${ }^{40}$ Igor Cubillo: "Circodelia: Para sorprender a quinceañeros", Efe Eme, febrero, 2004, p. 52.

${ }^{41}$ Serge Lacasse: "Intertextuality and Hypertextuality in Recorded Popular Music", The Musical Work: Reality or Invention?, Michael Talbot (ed.), Liverpool, Liverpool University Press, 2000, pp. 35-58.

42 Matthew Bannister: "'Loaded': Indie Guitar Rock, Canonism, White Masculinities", Popular Music, 25, 2006, pp. 77-95, 80-84.

${ }^{43}$ M. Bannister: "Loaded': Indie...., pp. 78-79, 82

${ }^{44}$ Ibid., p. 86.
} 
(como el "sonido Madchester" o los Smiths) o comerciales (como el synth pop de Depeche Mode o los Beatles), sino también por las implicaciones de falta de originalidad. Por ello, el grupo trata de legitimarse a través del archivalismo, defendiendo que su segundo disco es más variado puesto que han ampliado sus referencias musicales (así, no perciben los Beatles como un producto pop comercial sino como un clásico $)^{45}$.

\section{Glam, ironía e inautenticidad}

Otro de los elementos destacados de Circodelia es la utilización de la ironía, que les sirve como herramienta para el desafío de la masculinidad de forma similar a como lo hacía el glam rock de los setenta. Así, en su single de éxito Las chicas de las canciones realizan una crítica al estereotipo heterosexual de la masculinidad parecida a la del grupo Pulp en I'm a Man, estudiada por Jason Lee Oaskes ${ }^{46}$. El tema obtuvo gran difusión gracias a su videoclip, en el que la banda aparecía tocando en un club de estriptis. Doris Leibetseder en Queer Tracks: Subversive Strategies in Rock and Pop Music analiza la herramienta de la ironía y su posible utilidad o potencialidad para la transgresión de los roles de género. La ironía es un elemento que a menudo forma parte de la parodia y que pone en marcha la ambigüedad y la ambivalencia para dividir el mensaje en dos planos de significado: lo que el signo explicita y la idea contraria de lo que explicita. Esta herramienta resulta eficaz porque confirma lo que plantea la semiótica, que no es en el signo en sí donde está el significado, si no que éste se construye. Por lo tanto, la ironía revelaría que las identidades de género se forman mediante estructuras creadas artificialmente, pero naturalizadas a través de la repetición ${ }^{47}$.

Las chicas de las canciones contiene características musicales propias del glam rock (por ejemplo, coros onomatopéyicos), así como su androginia y su teatralidad. El texto alude de manera irónica a una serie de productos que se asocian con la masculinidad más convencional y estereotipada (coches deportivos, velocidad, fuerza, músculos, sexualidad agresiva.... $)^{48}$ añadiendo el rock a la lista al preguntarse repetidamente dónde están las atractivas groupies que se supone toda banda del género debe tener. La ironía

\footnotetext{
${ }^{45}$ I. Cubillo: "Circodelia: Para...".

46 Jason Lee Oakes: "I'm a Man': Masculinities in Popular Music", The Ashgate Research Companion to Popular Musicology, Derek Scott (ed.), Farnham, Ashgate, 2009, pp. 221-239.

${ }^{47}$ Doris Leibetseder: Queer Tracks: Subversive Strategies in Rock and Pop Music, Farnham, Ashgate, 2012, pp. 15-34.

${ }^{48}$ El texto de la tercera estrofa dice: "Uso lo más macho en after shave, / y en el ascensor me siguen dando la espalda. / Uso calzoncillos de Calvin Klein/ y tampoco me la noto más larga. / He fumado buen tabaco americano, / he guiado un deportivo muy caro. / Detrás del icono de la masculinidad, / me he gastado una pasta brutal".
} 
se encuentra en el hecho de que en su videoclip aparecen "chicas de las canciones", pero siempre separadas del grupo, por lo que no personifican una situación real, sino una fantasía o un mito.

Además, en el videoclip durante el solo de guitarra que precede al estribillo final, el cantante -que muestra una imagen andrógina, con maquillaje y ropa llamativa-interactúa con la barra de estriptis mientras su compañero imita los característicos gestos fálicos asociados con la performance de este instrumento. Así, esta performance demuestra que no solo no han aumentado su masculinidad al convertirse en una banda de rock, sino que además han perdido parte de la misma al exponerse ante el público de ambos sexos. Por lo tanto, Circodelia subrayan mediante la ironía que los gestos o signos no poseen significado propio, sino que este es añadido: no es el solo de guitarra de rock el que denota masculinidad -ya que, en su caso, sirve de acompañamiento para el estriptis del cantante- sino el discurso en el que este se desarrolla.

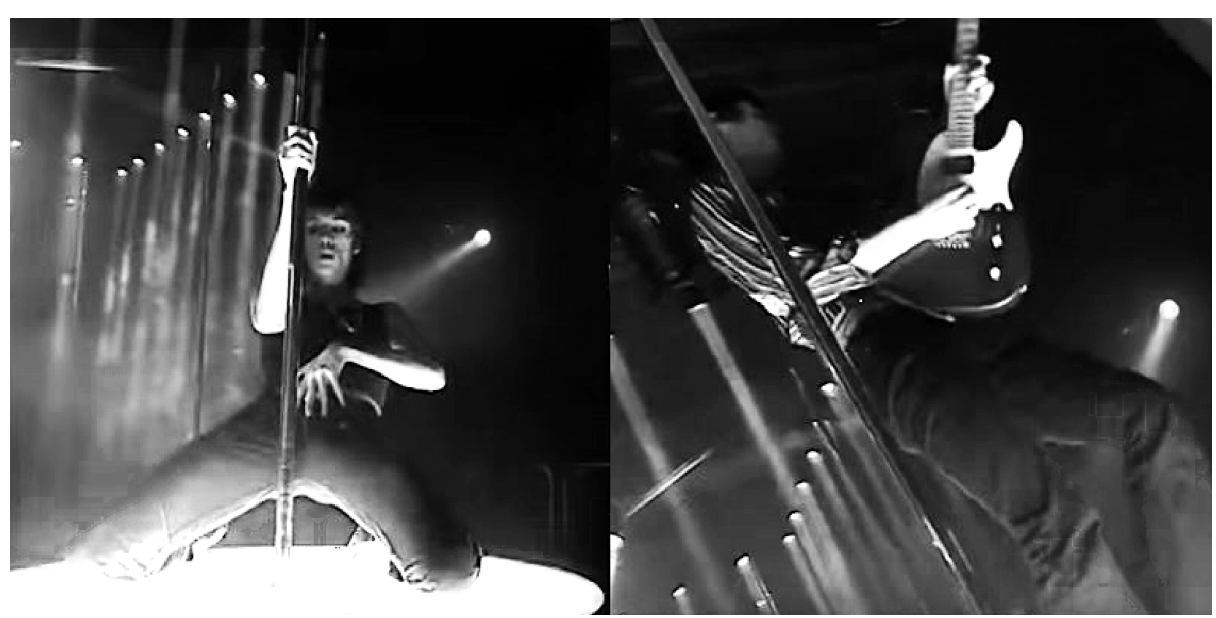

Fotograma del videoclip Las chicas de las canciones de Circodelia: Victor Pérez jugando con una barra de estriptis mientras Pablo Parser realiza el solo de guitarra $(3: 46 ; 3: 54)$

(https://www.youtube.com/watch?v=prdVeupkiEc. Última consulta: 6-6-2016)

Circodelia -como Babylon Chat- trataron de insertar su discurso dentro del revival del rocanrol clásico, pero su fidelidad a las estrategias del glam rock británico de los setenta supuso un problema. Así, el grupo suscribió las ideas sobre la autenticidad del rock para tratar de minimizar las connotaciones de inautenticidad heredadas del glam: condenaron, por ejemplo, el uso del playback y las aspiraciones a crear un superventas, ya que ambos hechos levantan sospechas de falta de profesionalidad y de alianza con la industria y el negocio del entretenimiento. Por otra parte, su adaptación del género no era un problema en la performance, ya que es precisamente la 
espontaneidad, energía y exceso del rock original a la que el glam retornaba lo que trataban de lograr:"Nuestro punto más revival está en el directo [...] sudamos la camiseta, llevamos equipo antiguo, ropa que brilla mucho, pantalones vaqueros muy ceñidos, maquillaje y pequeños ramalazos ambiguos. Es un show de rock" 49 .

El discurso andrógino de Circodelia - al contrario que el de Babylon Chatno solo ironizaba sobre la masculinidad, sino que incluía una imagen y unas actitudes asociadas con la feminidad (maquillaje natural y no grotesco, ir de compras, etc.) que los separaban de los estereotipos masculinos del rock -por ejemplo, en Peter $\mathrm{Pan}^{50}$ el cantante afirmaba tener "diez toneladas de ropa". De hecho, no fue la adaptación del glam la que dificultó su legitimación, sino haber escogido la vertiente del género (el glam británico de los setenta) que, a través de la adaptación de rasgos femeninos y el desafio irónico de la masculinidad tradicional, denotaba inautenticidad. Esto demuestra la versatilidad y el funcionamiento del glam, ya que es capaz de adaptarse a propuestas diferentes e incluso opuestas de androginia y masculinidad, manteniendo los rasgos que lo definen (la espectacularización y la performance del género).

\section{Los pecados eléktricos de Glamour to Kill: queer y retromanía}

Glamour to Kill surgieron a principios de la década del 2000 en Alemania a raíz de la colaboración entre Luis Miguélez (guitarrista ligado a la Movida) y Antonio Culebras (Antonio Glamour) ${ }^{51}$. Juntos comenzaron a abrirse hueco dentro de la escena underground de Berlín, donde residían, publicando el Ep Rock'n Roll Makes me Sexy en 2004 con la compañía Pale Music. Ese mismo año lanzaron Musik pour the ratas a través de la discográfica española Subterfuge Records y consiguieron difusión tanto en el extranjero como en España. En 2006 publicaron su segundo disco, Pecados eléctrikos (Subterfuge Records), ya como trío junto a Juan Tormento. Este trabajo y el anterior son los que obtuvieron mayor recepción, aunque siguieron en activo hasta el final de la década y lanzaron una extensión de Rock'n Roll makes me Sexy (Pale Music) en 2008 y un nuevo álbum, Creatures Without Soul (Pale Music), en 201052.

A menudo se consideró que la música de Glamour to Kill estaba dirigida al público homosexual y su líder Luis Miguélez trató de desmarcarse

\footnotetext{
${ }^{49}$ I. Cubillo: "Circodelia: Para...".

50 Penúltimo tema de su álbum Lo trágico es magnético (Pep’s Records, 2003).

51 Jose Pablo Jofré: “Exilio 'Fashion”, La Vanguardia, 4-II-2004, p. 29; Ángel Carmona: “Luis Miguélez. Glamour to Kill”, Efe Eme, marzo, 2006, p. 7.

52 Manuel Cuéllar: "Glamour to Kill: 'Lady Gaga está muy sobrevalorada", El País, 27-V-2011, http://cultura.elpais.com/cultura/2011/05/26/actualidad/1306360813_850215.html (última consulta: 17-1-2016).
} 
de dicha apreciación, achacándola a la supuesta poca apertura de la audiencia española hacia discursos como el de Boy George en los que los roles de género se trasgreden ${ }^{53}$. Sin embargo, la construcción de la autenticidad y de las identidades de género del grupo hace uso de la ambigüedad de tal forma que, homólogamente a lo que la teoría queer persigue, resulta difícil encasillar su propuesta dentro de una dicotomía. El estilo musical de Glamour to Kill ha sido etiquetado como "electro-punk", "glam-punk" o "post-punk irónico", entre otros $^{54}$. Lo cierto es que, en efecto, el grupo combina la fi-

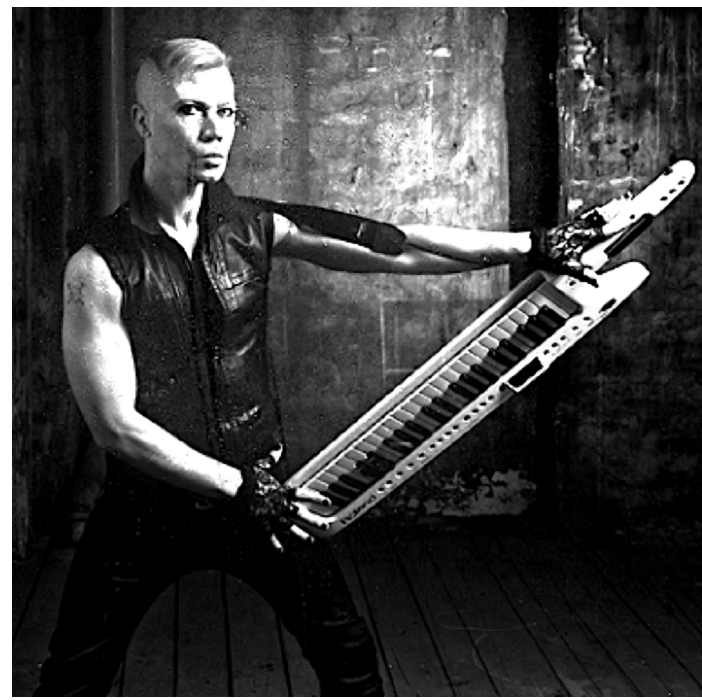

Juan Tormento, componente de Glamour to Kill, con un sintetizador con forma de guitarra

(Fuente: Gonzalo Armas, "Glamour to Kill", gonzaloarmas photography, http://www.gonzaloarmas.com/gtk.htm.

Última consulta: 11-4-2016)

losofia amateur de "un solo acorde" del punk ${ }^{55}$ con la electrónica retro de la new wave, caracterizada por el sonido de sintetizador y que, en casos como el de Gary Numan, trata de lograr la misma economía de recursos ${ }^{56}$. Glamour to Kill utilizan el sintetizador, por un lado, para enfatizar los power chords de la guitarra eléctrica, que expresan poder y que se asocian con la masculinidad ${ }^{57}$, y, por el otro, para introducir el baile, que connota ambigüedad $^{58}$. Asimismo, la guitarra y sus riffs son con frecuencia subrayados visualmente en los vídeos, así como sus connotaciones de fuerza al usar un sintetizador con la forma de este instrumento e imitar los gestos fálicos asociados con él.

Por lo tanto, no es posible identificar el estilo musical de Glamour to Kill -cuyas características básicas son la reinterpretación y el revival del punk y el synth pop-con un rol de género concreto, ya que incluye elementos que connotan masculinidad (power chords, guitarra eléctrica, punk) al tiempo

53 Á. Carmona: "Luis Miguélez..."

${ }^{54}$ J. P. Jofré: "Exilio 'Fashion"”, op. cit.

55 Dave Laing: One Chord Wonders: Power and Meaning in Punk Rock, Oakland, PM Press, 1985.

56 T. Cateforis: Are We..., pp. 69-70, 167.

${ }^{57}$ Robert Walser: Running with the Devil: Power, Gender and Madness in Heavy Metal Music, Hanover, University Press of New England, 1993, p. 2.

58 T. Cateforis: Are We..., p. 153. 
que la desestabilizan (baile, sintetizador, música disco). Esta indefinición queer es la que, al salirse de las clásicas dicotomías, provoca la suspicacia del público y la consecuente etiqueta de "música para gays" 59.

Las connotaciones de heterosexualidad presentes en el rock han sido estudiadas por diferentes investigadores, pero también su ambigüedad: así, se han observado características queer en su discurso, por ejemplo, en la figura de Elvis Presley ${ }^{60}$. Glamour to Kill usan el rock para atraer la mirada pública y desestabilizar, así, las identidades de género tradicionales en las que es la mujer el objeto que se observa: ello se puede apreciar en su tema Rock'n Roll makes me sexy, donde dejan claro que el rock es libertad para poner en juego su atractivo sexual -"Rock and roll makes me sexy / Rock and roll makes me free" ("El rock and roll me hace sexy / el rock and roll me hace libre"). El rock está simbolizado en la figura de la guitarra y su riff, que es subrayado visualmente en el videoclip -de hecho, es el único elemento rock de la canción-. Por lo tanto, el propio género del rock es para Glamour to Kill un componente queer más de su propuesta.

El deseo de incluirse dentro del rock hace que, además, enfaticen su carácter underground, quejándose de la incomprensión de su discurso en España. Así, su líder Luis Miguélez opina que la industria española es "absolutamente comercial y creativamente muy pobre [...] Para ellos el underground es sinónimo de no dinero [...] aquí [en Alemania] el underground vende". ${ }^{61}$ Del mismo modo, el grupo descalifica el discurso de Lady Gaga, dirigido a una audiencia pop y masiva: "Lo siento mucho por todos los fans, pero cualquier canción de Lady Gaga está absolutamente sobrevalorada. [...] me gusta su trasgresión. Pero musicalmente, no me aporta nada" 62 . Por otra parte, a pesar de que Glamour to Kill no recurren a sonidos del glam rock sí utilizan el glam como adjetivo estilístico al conservar elementos esenciales de su discurso (inclusión del baile, revival, artificialidad, espectáculo y ambigüedad). Su adaptación encaja, además, con el resto de su propuesta, ya que el glam fue la primera corriente queer del rock ${ }^{63}$.

Si la autenticidad y el género son construcciones performadas e intrínsecamente relacionadas, entonces el discurso queer de Glamour to Kill afecta a su percepción y aceptación por parte de las audiencias presumiblemente heterosexuales del rock. Sin embargo, el grupo se esfuerza por crear su versión de la autenticidad, asociándose, por ejemplo, con el underground o

\footnotetext{
59 Á. Carmona: "Luis Miguélez....".

${ }^{60}$ Freya Jarman-Ivens: "Don't Cry Daddy": the Degeneration of Elvis Presley's Musical Masculinity", Oh Boy!: Masculinities and Popular Music, Freya Jarman-Ivens (ed.), Nueva York, Routledge, 2007, pp. 161-180.

${ }^{61}$ J. P. Jofré: "Exilio 'Fashion"”, op. cit.

${ }^{62}$ M. Cuéllar: "Glamour to Kill...".

${ }^{63}$ D. Leibetseder: Queer Tracks:..., p. 6.
} 
demostrando actitudes asociadas con la masculinidad, como el poder o la capacidad de control. Así, en el videoclip de I Like Your boyfriend, cuyo texto manifiesta abiertamente una tendencia bisexual o indefinida -su letra repite de forma constante las frases "me gusta tu novio" ("I like your boyfriend"), "me gusta tu novia" ("I like your girldfriend"), sin llegar a decantarse por ninguna-, el trío aparece personificado en una mujer joven que tortura y utiliza a un hombre como objeto sexual. Esta simbiosis entre elementos masculinos de poder y violencia, y otros femeninos asociados con el glamour (pintalabios, tacones, etc.) es evidente en toda la producción del grupo - por ejemplo, la portada de su single Clone Fashion Remixes es una calavera de color magenta. De este modo, el discurso de Glamour to Kill es auténticamente queer (como manifiestan en su tema I'm a Queer64), ya que su estrategia es homogénea y sus ambigüedades son coherentes al centrarse todas en la disolución y desestabilización de las dicotomías tradicionales y hegemónicas (no son ni heterosexuales / ni homosexuales, ni auténticos / ni inauténticos, ni femeninos / ni masculinos).

\section{El caso de Nancys Rubias: el revival de la auto-prefabricación}

La banda española Nancys Rubias incluye el glam y absorbe los revivals de la década del 2000 a través de un discurso paródico y ecléctico que desafia, mediante elementos del camp y del kitsch, los estereotipos de género y las jerarquías de la cultura popular. Se formaron en 2004 y sus componentes son Mario Vaquerizo y su hermana Marta, Juan Pedro del Moral, Miguel Balanzategui y Susie Pop. Después de actuar en la sala Morocco fueron contratados por la compañía Warner, que había absorbido a la independiente $\mathrm{DRO}$, para elaborar varios discos en que la mayoría de las canciones están compuestas por Nacho Canut (componente de Fangoria) y producidas por Juan Carlos Moreno. Su primer disco, Nancys Rubias (DRO EastWest, Warner Music), se lanzó en 2005, tras lo cual el grupo comenzó a realizar conciertos como teloneros de Fangoria, junto a los que participaron en la celebración del día del Orgullo Gay en Madrid en 200765. En 2007 lanzaron Gabba Gabba Nancy (DRO) y en 2008 grabaron su tercer álbum Una cita con Nancys Rubias (WEA), cuya promoción se vio interrumpida por el suicidio de su componente Susie Pop, a pesar de lo cual el grupo no se disolvió. Además, la MTV España empezó a difundir en 2011 el programa de telerrealidad Alaska y Mario que Vaquerizo realiza junto a su mujer Olvido Gara (Alaska), lo que dio visibilidad a la banda a nivel nacional. Grabaron entonces su cuarto disco Ahora o nunca cuyo single

${ }^{64}$ Queer está incluido en Rock'n Roll Makes Me Sexy (Pale Music, 2004).

65 Â. Carmona: "Nancys Rubias: Muñecas divinas", Efe Eme, abril, 2005, 14. 
Peluquitas logró alcanzar el éxito. En 2012 publicaron el recopilatorio Nancys Hits (DRO), que contiene como extra un villancico navideño, El mejor regalo eres tú (All I Want for Christmas Is You). Actualmente son uno de los grupos más conocidos a nivel nacional, continuando en activo con éxito.

Nancys Rubias asimilan el glam -al igual que hacían Pedro Almodóvar y Fabio McNamara en los ochenta- en relación con las ideas de Warhol y la posmodernidad, que en la primera década del siglo XXI se entienden como un revival. Lawrence Grossberg explica que la sensibilidad posmoderna se caracteriza por la lógica de un "nihilismo irónico" o "auténtica inautenticidad" 66 , la cual conecta con la estética del "arte anti-arte" del pop $\operatorname{art}^{67}$ : ambas tratan de lograr legitimidad mediante la irónica conciencia de la naturaleza construida y artificial de sus textos, la cual no todo el público, sino sólo cierta "élite", sería capaz de percibir. Estos dos discursos (la posmodernidad y el "arte anti-arte" de Warhol) comparten ciertas estrategias con el glam rock de los setenta (incorporación de los mass media, rechazo de la autenticidad, pérdida del aura, etc.), que, en esencia, trasladaba las ideas de Warhol al contexto del rock y del pop ${ }^{68}$.

Nancys Rubias revisan ambos discursos a través del glam, que posibilita su inclusión en el contexto mediático musical para mostrar conciencia sobre su propia condición de producto de consumo y, por tanto, de la falsedad o artificialidad de sus pretensiones artísticas. Así, hacen uso manifiesto del playback para evidenciar que para ser una estrella del pop o del rock no es necesario tener habilidades musicales, sino -como ya explicaban Pedro Almodóvar y Fabio McNamara-, saber actuar. Por lo tanto, Nancys Rubias son en parte un revival del discurso propuesto por Almodóvar \& McNamara dentro de la Movida, adaptado al concepto de banda de rock (por ello, Mario Vaquerizo afirma que ellos son "el primer grupo auto-prefabricado del pop español" ${ }^{\prime 9}$ ).

\section{"Glamazonia": el camp y el kitsch en Nancys Rubias}

El camp y el kitsch siempre han estado relacionados, pero el uso masivo del revival en estos últimos años ha estrechado sus puntos de conexión. El término kitsch se utiliza para aludir a un tipo de estética considerada de mal gusto por la estereotipación y la falsificación de los valores positivos más evidentes de un objeto / obra. La sensibilidad camp, por su parte, es un

\footnotetext{
${ }^{66}$ L. Grossberg: We Gotta..., pp. 224-234.

${ }^{67}$ Matthew Bannister: White Boys, White Noise: Masculinities and 1980s Indie Guitar Rock, Aldershot, Ashgate, 2006, pp. 37-44.

${ }^{68}$ Van M. Cagle: Reconstructing Pop / Subculture: Art, Rock and Andy Warhol, Thousand Oaks, Sage Publications, 1995.

69 Á. Carmona: "Nancys Rubias: Muñecas divinas", Efe Eme, abril, 2005, p. 14.
} 
tipo de apreciación irónica que se realiza a través de la exageración y de la evidencia de artificialidad. La ironía del camp originalmente se hallaba conectada con la transgresión de género, ya que eran los artistas involucrados en los ambientes homosexuales quienes, a través del refinamiento estético y el dandismo, eran capaces de percibirla. Sin embargo, el camp se ha incorporado a la cultura popular como capital cultural e indicación de buen gusto y cosmopolitismo, por lo que su capacidad de transgresión puede haberse limitado al convertirse él mismo en una falsificación kitsch del originado en los círculos homosexuales ${ }^{70}$.

Los artistas que adaptaron el glam al contexto español de los ochenta (Almodóvar \& McNamara,Alaska, Tino Casal) pertenecían al círculo de los pintores Las Costus -Juan Carrero (1955-1989) y Enrique Naya (19531989)-, quienes crearon su propia versión española del camp y del pop art $t^{71}$. Por ejemplo, la colección de cuadros El Valle de los Caídos (1980-1987) responde a este tipo de sensibilidad al adaptar de manera irónica estereotipos de la pintura barroca y sustituirlos por iconos de la sociedad de consumo como la cantante pop Alaska ${ }^{72}$. De este modo, el uso del camp por Nancys Rubias conecta con los artistas de la Movida que ya habían adaptado esta sensibilidad estética a la música pop a través del glam.

Nancys Rubias desarrollan su discurso en un momento en el que la autenticidad del rock y los elementos implicados en su construcción (como la masculinidad o la profesionalidad artística) están tan estereotipados y falseados por los medios que se podrían considerar kitsch. Así, utilizan el camp para bromear sobre este mito aludiendo irónicamente a géneros auténticos y masculinos, como el rocanrol de los cincuenta o el punk, reutilizando iconos cuya artificialidad ya era evidente o bien por su exceso -los vídeoclips de No estás curada y Corazón de hielo están rodados en las Vegas (ciudad ligada a la iconografia de la última etapa de Elvis, en donde las características espectaculares del rock se hicieron más evidentes) - o por su componente glam -la portada de Nancys Rubias (2005) y de Gabba Gabba Nancy (2007) son imitaciones de los álbumes New York Dolls (1973), de los New York Dolls, y Flaunt it (1986), de Sigue Sigue Sputnik, respectivamente.

\footnotetext{
${ }^{70}$ Fabio Cleto: "Introduction: Queering the Camp", Camp: Queer Aesthetics and the Performing Subject, Fabio Cleto (ed.), Ann Arbor, University of Michigan Press, 1999, pp. 1-42; Ruth Holliday, Tracey Potts: "Camp kitsch", Kitsch!: Cultural Politics and Taste, Manchester, Manchester University Press, 2012, pp. 115-153.

${ }_{71}$ Teresa Vilarós: El mono del desencanto: una crítica cultural de la transición española (1973-1993), Madrid, Siglo XXI, 1998, pp. 263-265; Alberto Mira: De Sodoma a Chueca. Una historia cultural de la homosexualidad en España en el siglo XX, Barcelona, EGALES, 2004, pp. 516-521.

72 Alaska pintada como La Piedad por los Costus en 1986. Sobre Costus-Coútus: http://www.costus.es/portfolio/valle-de-los-caidos/ (última consulta: 25-4-2016).
} 


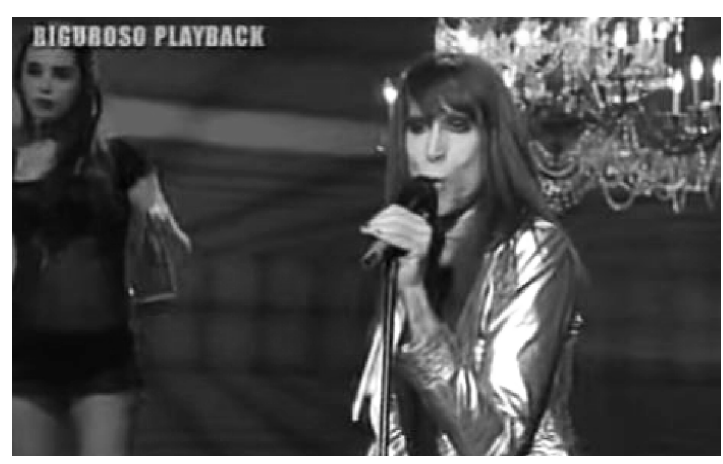

Las Nancys Rubias en el programa Carta Blanca interpretando Maquíllate con el rótulo "riguroso playback" (2:57) (https://www.youtube.com/watch?v=Io9bbHDBDI8. Última consulta: 9-5-2016)
Además, Nancys Rubias ironizan sobre la inautenticidad posmoderna, que también se ha convertido en un estereotipo, parodiando la utilización manifiesta de elementos como el playback: por ello, incluyen en pantalla el rótulo "riguroso playback" para imitar el habitual "riguroso directo" que prueba irónicamente, ya que para el espectador ambas comprobaciones (tanto el directo, como el playback) son técnicamente imposibles-, la interpretación en televisión de los artistas. De esta forma, Nancys Rubias reutilizan el mecanismo a través del que los grupos de rock aseguran normalmente su autenticidad (afirmando ser ellos los que tocan) para certificar su inautenticidad.

Emily Dolan explica que los revivals del s. XXI han comprometido el carácter de mal gusto y de falsificación del kitsch al percibir cierta autenticidad en la revisión de estos objetos ya que al pertenecer estos al pasado poseerían un aura sentimental generada por la nostalgia ${ }^{73}$. Así, las reutilizaciones hechas por Nancys Rubias desafian los cánones tradicionales de la cultura popular al permitir la autentificación tanto de iconos underground -Sigue Sigue Sputnik-, como mainstream -el tema Abre tu mente es una versión del éxito de la cantante pop Merche-e incluso de productos pop desprestigiados o kitsch -el recopilatorio Nancys Hits (2012) incluye un villancico y está diseñado imitando la revista Súper Pop de iconos juveniles- que contendrían dicho aura por asociarse con el pasado (villancico/ infancia; fan/ adolescencia).

Asimismo, el propio glam ha sufrido un proceso similar al del camp al ser incorporado, explotado y estereotipado dentro de los medios masivos. Sin embargo, la permeabilidad y versatilidad de sus elementos de transgresión permiten que Nancys Rubias parodien y (auto) critiquen esta "falsificación" del género mientras lo ponen en práctica: por ejemplo, titulan Glamazonia a uno de sus temas ${ }^{74}$ para exagerar e ironizar sobre la utilización reiterada de elementos "selváticos", como estampados de leopardo, boas de plumas, etc., dentro del género.

${ }^{73}$ Emily I. Dolan: “.... This Little Ukulele Tells the Truth': Indie, Pop and kitsch Authenticity”, Popular Music, 29, 2010, pp. 457-469: 465-466.

74 Glamazonia está incluido en Una cita con Nancys Rubias (WEA, 2009). 


\section{"Supertravesti": camp e identidades género}

El nombre de Nancys Rubias hace alusión a los New York Dolls ("las muñecas de Nueva York”), quienes según Van M. Cagle constituían una parodia camp del glam $^{75}$. Cada uno de sus componentes se identifica con un tipo de muñeca Nancy -Nancy Anoréxica (Mario Vaquerizo), Nancy Travesti (Juan Pedro del Moral), La Verdadera Nancy Rubia (Susie Pop), Nancy O (MartaVaquerizo) y Nancy Reagan (Miguel Balanzategui)-, pero adoptan su rol de manera personal, por lo que su estrategia estaría cercana a la de la mascarada ${ }^{76}$. Asimismo, la parodia es una imitación que -al contrario que el pastiche, que es neutral- introduce la distancia crítica y utiliza la ironía para generar dobles sentidos: a través de esta repetición podemos subrayar ciertos elementos diferenciadores del original, para hacer ver que este no es espontáneo o natural, sino que está construido ${ }^{77}$.Así, esta estrategia permite a Nancys Rubias evidenciar que las identidades de género están tan performadas y calculadas como su puesta en escena "autoprefabricada".

Por ejemplo, el grupo parodia el estereotipo de la masculinidad y la homosocialidad del rock utilizando el punk para reivindicar el uso del maquillaje en Maquíllate y cambiar el tradicional "sexo, drogas y rocanrol" por "lujo, drogas, liposucción". De esta forma, Nancys Rubias ponen de manifiesto que los hombres invierten tanto tiempo en fabricar su estereotipada masculinidad (por ejemplo, involucrándose en una banda de rock) como las mujeres en su feminidad (identificada, irónicamente, con la superficialidad y la excesiva preocupación por la belleza física). Nancys Rubias parodian, además, los estereotipos femeninos del rock mediante la única mujer del grupo (Marta Vaquerizo) que representa una ironía del estereotipo pin-up de "mujer objeto" al tocar un instrumento totalmente banal -el triánguloque ni siquiera suena en la mayoría de las canciones, por lo que, en sentido estricto, ni siquiera se puede considerar que está haciendo playback.

Finalmente, la banda hace ver con su discurso que también la ambigüedad sexual se ha convertido en un estereotipo kitsch del rock: así, Mario Vaquerizo exagera y teatraliza el afeminamiento del rol del cantante -por ejemplo, en su actuación en Crónicas Marcianas en 2005 no solamente utiliza tacones de mujer y maquillaje, sino que además exhibe una camiseta con el nombre de Elvis (en referencia a Elvis Presley) en purpurina $\operatorname{rosa}^{78}$.

75 V. M. Cagle: Reconstructing Pop..., p. 193.

${ }^{76}$ D. Leibetseder: Queer Tracks..., pp. 92-95.

77 Ibid., pp. 35-58.

78 Nancys Rubias: "Nancys Rubias, Nancys Rubias (Actuación en CM)", Crónicas Marcianas, Telecinco, 2005, https://www.youtube.com/watch?v=zQ7DuUtyllo (última consulta: 30-1-2016). 


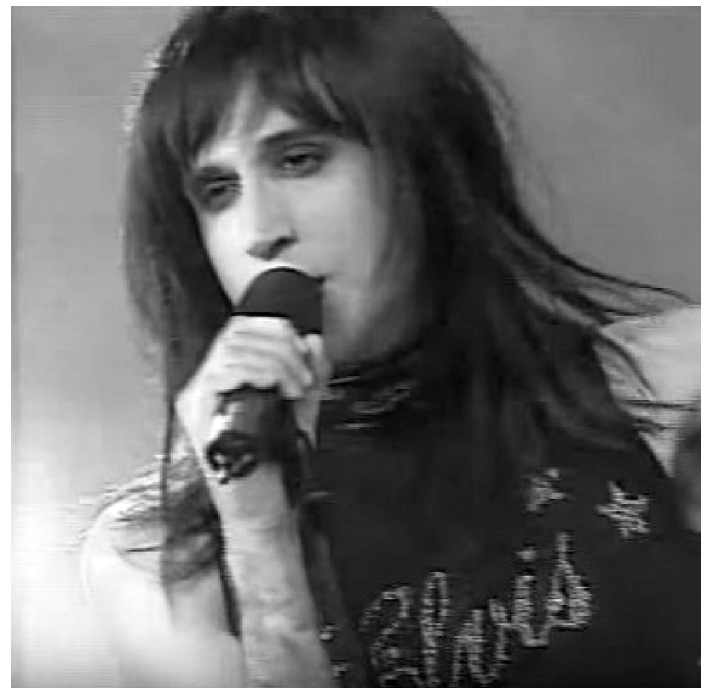

Mario Vaquerizo, líder de Nancys Rubias, con una camiseta de Elvis Presley con purpurina rosa para su actuación en

Crónicas Marcianas en 2005

Como los New York Dolls, Nancys Rubias evidencian, a través de la exageración irónica del camp, que los súper-modelos (o "Súpertravestis"79) de la transgresión (Mick Jagger, David Bowie, Boy George, ... Elvis Presley) están tan asimilados dentro de los mass media como el resto: Elvis ya no es un sólo un icono, sino la marca industrial del rock y su ambigüedad.

\section{Conclusiones}

Los revivals del glam en la primera década del 2000 en España se entrecruzan en el proceso general de retromanía, que se volvió más rápida y ecléctica por la masificación de Internet. La heterogeneidad del glam -entendido como elemento adaptable a diferentes contextos- le permitió insertarse en diferentes revivals sin perder la capacidad de ser reconocido (el adjetivo glam sigue funcionando como indicador de la ambigüedad de género y la espectacularización). Por ello, la amplia presencia e hibridación de los discursos del glam en el fenómeno de la retromanía no es casual, sino consecuencia de su particular versatilidad por haber sido el primer revival del rock ${ }^{80}$.

Los grupos que he analizado, a pesar de su diversidad y de absorber influencias diferentes, usan el glam para performar y/o transgredir las identidades sexuales (heterosexualidad, homosexualidad, bisexualidad) y los roles de género (feminidad, masculinidad, androginia). Las herramientas de estos revivals (ironía, camp, parodia, etc.) ni son las mismas ni están incorporadas de igual manera, pero todos las utilizan para demostrar que el género no es una categoría biológica, sino una construcción performada tan artificialmente como el propio glam: así, Babylon Chat utilizó el glam para volver al rocanrol y construir su autenticidad, ligada a una performance de la

\footnotetext{
79 Supertravesti es un tema incluido en Gabba Gabba Nancys (DRO, 2007).

80 "Retro thus becomes a structural feature of pop culture [...] In music, the first of these slowdowns occurred in the early seventies. [...] the first backwards-looking movement within rock, glam (...)". S. Reynolds: Retromania: Pop..., p. 197.
} 
heterosexualidad; Circodelia presentó una propuesta ecléctica que adaptaba la androginia y la ironía del glam rock británico de los setenta, del que heredaron su inautenticidad, como parte del canon de la música popular; Glamour to Kill recurrió al uso del sintetizador y al propio género del rock para construir un discurso queer que eludía las dicotomías tradicionales; y, por último, Nancys Rubias utilizaron la sensibilidad camp y la estética kitsch para evidenciar la estereotipación tanto de la autenticidad y de la inautenticidad, como de las identidades de género en el pop y el rock.

Por último, cabe señalar que, más allá de la utilización del idioma castellano, no existen rasgos españoles reconocibles dentro de estas cuatro propuestas, que poseen muchas similitudes con sus modelos foráneos: y es que ni importa ni probablemente los grupos se plantearon este tipo de discurso de la diferencia. No obstante, el glam español y el anglosajón se crean dentro de un contexto cultural similar, donde la masculinidad heterosexual es la identidad dominante. Por lo tanto, el paralelismo entre sus estrategias no es solo consecuencia de la imitación, sino de que tanto los grupos españoles como los anglosajones han de lidiar con construcciones de género similares, que han impregnado el rock desde sus inicios.

Recibido: 25-5-2016

Aceptado: 8-9-2016 